\title{
Dynamics and spectroscopy of molecular ensembles in a lossy microcavity
}

Inga S. Ulusoy ${ }^{1, \text { a) }}$ and Oriol Vendrell ${ }^{1, b)}$

Theoretical Chemistry, Institute of Physical Chemistry, Heidelberg University, Im Neuenheimer Feld 229, 69120 Heidelberg, Germany

(Dated: 1 July 2020)

The radiative and nonradiative relaxation dynamics of an ensemble of molecules in a microcavity are investigated with emphasis on the impact of the cavity lifetime on reactive and spectroscopic properties. Extending a previous study [J. Phys. Chem. A 123, 8832 (2019)], it is shown that the dynamics of the ensemble and of single molecules are influenced by the presence of a cavity resonance as long as the polariton splitting can be resolved spectroscopically, which critically depends on the lifetime of the system. Our simulations illustrate how the branching between nonradiative intersystem crossing and radiative decay through the cavity can be tuned by selecting specific cavity photon energies resonant at specific molecular geometries. In the case of cavity-photon energies that are not resonant at the Franck-Condon geometry of the molecules, it is demonstrated numerically and analytically that collective effects are limited to a handful of molecules in the ensemble.

\footnotetext{
a)Electronic mail: inga.ulusoy@pci.uni-heidelberg.de
}

b)Electronic mail: oriol.vendrell@pci.uni-heidelberg.de 


\section{INTRODUCTION}

The presence of resonances between a material excitation and a quantized electromagnetic mode results in the formation of hybrid light-matter systems. Because the resonance condition strongly depends on the nuclear positions of the material part, the photophysics and photochemistry of the hybrid system can be modified within properly tuned resonance microcavities.$^{1-9}$ This has lead to the proposal, development and improvement of technological applications at the crossroads of chemistry, materials science and photonics, with examples ranging from energy and charge transport in the material part ${ }^{7,10-13}$ to applications in which the photonic characteristics of the hybrid system play the prominent role. ${ }^{14-17}$

The theoretical description of polaritonic systems has rapidly evolved in the past years, from treating the material part as a two-level system (atom) in the framework of the Jaynes-Cummings ${ }^{18}$ or Tavis-Cummings ${ }^{19}$ Hamiltonian, to including molecular Hamiltonians via i.e. polaritonic potential energy surfaces ${ }^{20}$ (pPES) or in the basis of the field-free rovibronic states for spectroscopic properties, ${ }^{21}$ quantum electrodynamics density functional theory ${ }^{22,23}$ (QEDFT), extensions of the exact factorization approach ${ }^{24-26}$ and approaches based on wave-packet propagation methods. ${ }^{8,9,27-29}$ However, a detailed understanding of the interplay of various decay channels involving cavity losses and the multidimensional quantum dynamics of nuclei in single molecules and ensembles is mostly missing. In particular, the characterization of the spectroscopic response of the systems interacting with external light and its connection to the underlying dynamics is required to probe phenomena such as energy dissipation and decoherence. This is crucial for the development of photonic devices ${ }^{1}$ and the development of chemical and physical applications. ${ }^{2,4,5}$

Radiative cavity decay - leakage of photons to the electromagnetic continuum modes - can be approached through dissipative terms in the theoretical description. The dissipative dynamics can be treated phenomenologically via decay rate constants, for example in the framework of density-matrix propagation, ${ }^{30-32}$ in combination with molecular dynamics ${ }^{33,34}$ or through a master equation approach based on the Lindblad form, ${ }^{35-37}$ to name a few. The cavity decay can only take place from the polaritonic states that have a significant optical component, the upper polaritonic (UP) and lower polaritonic (LP) states. The dark state polaritonic (DSP) states cannot directly emit photons; first a decay to either the LP or UP states must take place. ${ }^{30,34,35,37-40}$ The effective cavity decay rate decreases for polaritonic states, compared to the bare cavity; in some cases this may be a consequence of the population transfer to DSP and mediated by nonadiabatic effects. However the 
delayed emission from the LP state observed in some experiments is not fully explained yet. ${ }^{41-45}$ For example, it has been discussed controversially in the literature whether the LP decay constant reflects the DSP to LP decay as the bottleneck in the dynamics, rather than the LP decay. ${ }^{4,46,47}$

The cavity decay, through the cavity lifetime, also plays an important role in the definition of weak vs. strong coupling. ${ }^{48}$ In theoretical studies, "strong" coupling is often merely defined as the Rabi splitting being a significant fraction of the cavity excitation energy $\omega_{C}$. More precisely though, "weak" and "strong" coupling can be differentiated by the spectral resolution of the LP/UP splitting. In weak coupling, the splitting is smaller than the spectral linewidth and thus hidden in the spectrum; in strong coupling, the splitting is clearly visible. Thus, not only the magnitude of the Rabi splitting, but also the linewidth of the involved states determines the classification as strong coupling, and consequently the energy splitting can amount to a significant fraction of the excitation energy but may not be observable in the spectrum due to a short cavity or matter decay time. ${ }^{49,50}$

Since the classification of the coupling regime is directly linked with the spectral separability of the upper and lower polaritonic states, one can ask whether this fact has consequences for the underlying dynamics of the hybrid system upon light absorption. We investigate this question by quantum dynamics simulations of $\mathrm{NaI}$ and pyrazine molecules in a cavity using the multiconfiguration time-dependent Hartree (MCTDH) approach ${ }^{51,52}$ including cavity decay to the continuum. We build on our previous work ${ }^{9}$ where we demonstrated that the photoinduced dynamics are dominated by the molecular deactivation pathways upon excitation into the LP mode, whereas the dynamics initiated in the UP mode are significantly more complex. It is shown that the first point only holds true for a cavity-molecule resonance at the Franck-Condon point. The impact of different cavity decay rates on the molecular dynamics and spectroscopic properties is studied, and we further investigate the role of the DSP in the radiative and nonradiative decay channels. Finally, we consider the tuning of the resonance condition of the cavity mode for different points of the configurational space of the molecules in foresight of applications in photon harnessing and frequency conversion.

In the following, the underlying theoretical approach is introduced, followed by the results on the $\mathrm{NaI}$ and pyrazine, their discussion and a summary. 


\section{THEORY}

For $N$ molecules coupled resonantly to a cavity mode, the ensemble-cavity system is described by the Hamiltonian

$$
\hat{H}=\sum_{\kappa=1}^{N} \hat{H}_{\mathrm{mol}}^{(\kappa)}+\hat{H}_{\mathrm{cav}}+\hat{H}_{\mathrm{las}}
$$

with $\hat{H}_{\mathrm{mol}}^{(\kappa)}$ the Hamiltonian for the $\kappa$-th molecule, $\hat{H}_{\mathrm{cav}}$ the cavity and cavity-molecule coupling Hamiltonian, and $\hat{H}_{\text {las }}$ the laser field (treated classically). The cavity mode is described as a single quantized electromagnetic mode with a constant electric field amplitude across all members of the molecular ensemble and a lifetime $\tau$ of the cavity photon that is related to the cavity decay constant through $\Gamma=1 / \tau$ and cavity quality factor $Q=\omega_{C} / \Gamma$ :

$$
\hat{H}_{\mathrm{cav}}=\left(\hbar \omega_{C}-i \frac{\Gamma}{2}\right) \hat{a}^{\dagger} \hat{a}+g \vec{\varepsilon}_{c} \cdot \overrightarrow{\hat{D}}\left(\hat{a}^{\dagger}+\hat{a}\right)+\frac{1}{2}\left(g \vec{\varepsilon}_{c} \cdot \overrightarrow{\hat{D}}\right)^{2}
$$

where $\vec{\varepsilon}_{c}$ is the cavity mode polarization direction, $\overrightarrow{\hat{D}}=\sum_{\kappa}^{N} \vec{\mu}^{\kappa}$ the dipole operator for the $N$ molecules with dipole moment $\vec{\mu}$, and $g=\sqrt{\frac{\hbar \omega_{C}}{2 V \varepsilon_{0}}}$ the coupling strength of the electromagnetic mode or cavity-mode field strength. In Eq. (2) the cavity is described by a complex energy, in which the imaginary part is proportional to the decay rate $\Gamma$ and which results in a non-Hermitian cavity Hamiltonian. ${ }^{53,54}$ Note that the zero-point energy of the cavity needs to be subtracted to avoid the nonphysical decay of the cavity ground state (i.e. $\left.\left(\hat{a}^{\dagger} \hat{a}+1 / 2\right) \rightarrow\left(\hat{a}^{\dagger} \hat{a}\right)\right)$. It is also readily seen that, under Hamiltonian (2), the decay rate of the cavity state $|N\rangle$ with $N$ photons is $N \Gamma$. This matches the physical picture that the probability per unit time for one of the $N$ photons to leak out of the cavity is $N$ times larger than for a single photon. The complex energy term in the Hamiltonian leads to a decrease of the wave function norm over time when the cavity excited state(s) is (are) populated. The decrease of the norm directly corresponds to the probability that the initial excitation of the system caused by external light spontaneously decays into the electromagnetic continuum. In other words, it corresponds to the radiative loss of the hybrid system mediated by the cavity mode, and is referred to as $P_{\mathrm{em}}$ in the following.

The portion of the wave function that has emitted a photon into the continuum states is no longer present in the dynamics simulation: The situation where the cavity-molecule ensemble carries no optical excitation, but the continuum states of the field outside of the cavity are excited, lies outside of the Hilbert state spanned by the wave function. For a more encompassing description of the cavity leakage to the continuum, not only the dissipation would need to be included in the 
Hamiltonian, but also fluctuation. In the Schrödinger description, this could be achieved by i.e. including a photon bath or using a stochastic approach and will be the target of future studies. At this point, the cavity decay will be described using the heuristic approach introduced above, which only considers the manifold where the vacuum field carries no excitation, and does not allow a description of the portion of the system after photon leakage.

At this point, for reasons of simplicity and generality, we consider only the case with one cavity-mode being resonant with a transition in the molecule; in a more general experimental setting, more than one cavity mode could couple to the molecules, leading to prolonged cavity leakage.

The laser interaction term reads

$$
\hat{H}_{\text {las }}=-\vec{E}(t) \cdot \vec{D}
$$

with electric field $\vec{E}(t)=\vec{\varepsilon}_{L} A(t) \cos \left(\omega_{L} t\right)$, where $\vec{\varepsilon}_{L}$ is the laser field polarization direction, $A(t)$ is the electric field envelope and $\omega_{L}$ the carrier frequency of the laser. Without loss of generality we make the model assumption that the laser field couples directly to the molecules and not to the cavity. ${ }^{8}$ The length gauge and the dipole approximation are used for both the quantized cavity mode and the laser field.

\section{MOLECULAR HAMILTONIAN}

The molecular Hamiltonian for the $\kappa$-th molecule in the ensemble reads

$$
\hat{H}_{\mathrm{mol}}^{(\kappa)}=\hat{T}_{n}^{(\kappa)}(\mathbf{Q})+\hat{H}_{e}^{(\kappa)}(\mathbf{r} ; \mathbf{Q})
$$

where $\hat{T}_{n}^{(\kappa)}(\mathbf{Q})$ is the kinetic energy operator for the nuclear degrees of freedom (dof) and $\hat{H}_{e}^{(\kappa)}(\mathbf{r} ; \mathbf{Q})$ consists of the electronic kinetic energy operator and the electron-electron, nucleielectron and nuclei-nuclei Coulombic terms. For NaI, an eigenstate basis is used based on ab initio potential energy surfaces (PESs) and dipole matrix elements. ${ }^{8,27}$ The molecular Hamiltonian for $\mathrm{NaI}$ reads

$$
\hat{H}_{\mathrm{NaI}}=\hat{T}_{n}(R) \mathbf{1}+\left(\begin{array}{cc}
V_{0}(R) & 0 \\
0 & V_{1}(R)
\end{array}\right)
$$

with $R$ the interatomic distance, and $V_{0}(R)$ and $V_{1}(R)$ the adiabatic potentials for the electronic ground state $S_{0}$ and excited state $S_{1}$. The identity is two-dimensional, so that the kinetic energy operator is identical for the two electronic states. 
The Hamiltonian used for pyrazine is a four-dimensional $\left(n_{\text {modes }}=4\right)$ vibronic coupling Hamiltonian to first order ${ }^{55,56}$ within the group Born-Oppenheimer approximation with respect to the molecular electronic states. ${ }^{57}$ The expansion is performed around the Franck-Condon (FC) point in terms of normal modes $Q_{i}$ :

$$
\begin{aligned}
\hat{H}_{\text {pyr }} & =\sum_{i=1}^{n_{\text {modes }}} \frac{\omega_{i}^{2}}{2}\left(-\frac{\partial^{2}}{\partial Q_{i}^{2}}+Q_{i}^{2}\right) \mathbf{1}+\Delta+\sum_{i \in\{\text { tun }\}}^{n_{\text {tun }}} \mathrm{K} Q_{i} \\
& +\sum_{i \in\{\text { coupl }\}}^{n_{\text {coupl }}} \Lambda Q_{i}
\end{aligned}
$$

for the three relevant electronic states $S_{0}, S_{1}$ and $S_{2}$, where the identity is now three-dimensional. The vibrational modes are separated into the set of coupling $\{$ coupl $\}$ and tuning $\{\operatorname{tun}\}$ modes, with the coupling mediating the nonadiabatic population transfer between $S_{2}$ and $S_{1}$; and the tuning modes leading to effective shifts of the potential minima. The first tuning mode, mode $v_{6 a}$, steers the molecule towards the conical intersection on both $\mathrm{S}_{2}$ and $\mathrm{S}_{1}$; whereas the other two tuning modes lead to an effective dephasing of the wave packet, as the level of vibrational excitation in $S_{1}$ and $S_{2}$ differs for these two modes, leading to a spread of the wave packet and moving the nuclei in different directions on the two PESs. The vibrational frequencies $\omega_{i}$ of the four modes $i$, and the coupling and tuning parameters $\Lambda$ and $\mathrm{K}$, as well as the energy shifts $\Delta$ between electronic states are the same as previously. ${ }^{9,55,56}$

\section{A. Quantum dynamics propagation and analysis}

The time-dependent Schrödinger equation for the molecular ensemble-cavity wave function is propagated using the MCTDH method ${ }^{51,52}$ and its multilayer generalization ${ }^{58-60}$ with the Heidelberg MCTDH package ${ }^{61}$, as introduced previously. ${ }^{27}$

After the hybrid cavity-ensemble system absorbs one photon, different final outcomes are possible. Either the cavity can emit the photon through radiative decay, or the molecules can retain the excitation and undergo a photophysical process or photochemical transformation - e.g. a photodissociation reaction ( $\mathrm{NaI}$ ) or internal conversion (IC, pyrazine). In both cases, the definition of product formation is straightforward: For NaI, we define that the molecule has dissociated when the interatomic distance exceeds $R_{d}=15 a_{0}$; so that the cumulative probability of dissociation $P_{\text {diss }}$ is directly related to the portion of the wave packet that occupies the grid at $R_{d}$ and larger. For pyrazine, the nonadiabatic electronic relaxation proceeds from $S_{2}$ towards $S_{1}$, and the population 
of $\mathrm{S}_{1}, P_{\mathrm{S}_{1}}$ thus directly corresponds to the cumulative probability of the IC process.

In this work, we are interested in the dynamics within the single excitation space, although we note that there is no methodological restriction in our approach to treat the dynamics in higher excitation spaces. When the dynamics are initiated using a laser pulse, the probability that the system is still in the ground state, $P_{\mathrm{GS}}$, is obtained by projecting the total wave function onto the ensemble's ground state. The overall excitation probability of the cavity-ensemble system needs to remain low to ensure that the dynamics proceeds in the single-excitation regime (one-photon absorption). A total excitation probability of at most $10 \%$ ensures that two-photon transitions remain roughly at a level of $1 \%$. The total excitation probability for the ensemble is given by $P_{\mathrm{ES}}=1-P_{\mathrm{GS}}$ and as different ensemble sizes are considered, the laser intensity is adjusted to keep the overall excitation probability approximately constant. This is achieved by scaling the laser intensity by $1 / \sqrt{N}$. It is assumed that the molecules absorb light independently. ${ }^{8}$

Alternatively, the initial state can be generated as a direct LP or UP excitation through application of the excitation operators $\hat{T}_{ \pm}$, which directly promote the cavity-molecule system to a pure upper (+) or lower (-) polaritonic state, or the $\mathrm{S}_{2}$ state:

$$
\begin{aligned}
& \hat{T}_{ \pm}=\frac{1}{\sqrt{2}}\left(\hat{a}^{\dagger}+\hat{a}\right) \mp \sum_{\kappa}^{N} \frac{1}{\sqrt{2 N}}\left(\left|0_{\kappa}\right\rangle\left\langle 1_{\kappa}\right|+\text { h.c. }\right) \\
& \hat{T}_{S_{2}}=\sum_{\kappa}^{N} \frac{1}{\sqrt{N}}\left(\left|0_{\kappa}\right\rangle\left\langle 1_{\kappa}\right|+\text { h.c. }\right)
\end{aligned}
$$

In this case, the initial state is either a pure polaritonic state or the $\mathrm{S}_{2}$ state at the FC geometry, and no population remains in the ground state, simplifying the analysis. Indeed, Eq. (7) represents a modified form of a dipole operator designed to excite the system within the single excitation space in a state-selective manner. This permits to gain insight into the dynamics resulting from the initial population of either the LP or UP states, even if they are not spectroscopically resolved and therefore could not be addressed separately by an actual laser pulse.

The dynamics and properties of the different cases are partially discussed with the help of the corresponding $\mathrm{pPESs}^{8,20,62}$, which are defined as the eigenvalues of the Hamiltonian

$$
\hat{H}_{\mathrm{pPES}}=\hat{H}-\sum_{\kappa=1}^{N} \hat{T}_{n}^{(\kappa)},
$$

in the single-excitation (one-photon) subspace and with $\hat{H}$ the total Hamiltonian without an external laser pulse; $\hat{T}_{n}^{(\kappa)}$ is the nuclear kinetic energy operator of the $k$-th molecule. These polaritonic potentials are not employed in the wave packet propagation, but merely serve as a tool to visualize the concepts and key points of the analysis. 


\section{RESULTS AND DISCUSSION}

\section{A. NaI: A two-state example}

The NaI photodissociation dynamics in a cavity have been previously investigated by us and others $8,9,28,63,64$. NaI is stable in its ground state but dissociates upon photoexcitation. In this example, the cavity energy is chosen such that the cavity is in resonance with the energy difference between the minimum of the ground state $S_{0}$ and the vertical energy of the excited state $S_{1}$ (FC point). For each molecule in the cavity, two electronic states and one vibrational dof are included in the dynamics. The cavity-molecule coupling parameters are adjusted so that the splitting between upper and lower polariton mode amounts to $\Omega_{R}=0.13 \mathrm{eV}$, which is about $1 / 30$ th of the cavity excitation energy $\omega_{C}=3.81 \mathrm{eV}$, and is kept constant for different numbers of molecules in the cavity by scaling the cavity-molecule interaction strength. This scaling physically implies that the number density of molecules in the cavity remains constant as its number varies.

From the ground state minimum, the molecule can directly be excited into either the lower or upper polariton mode by application of the corresponding excitation operator $\hat{T}_{ \pm}$. Three different cavity decay constants are chosen, $\Gamma_{f}, \Gamma_{m}$ and $\Gamma_{s}$, corresponding to fast, moderately fast and slow decay (decay times $\tau_{f}=1.2 \mathrm{fs}, \tau_{m}=12.1 \mathrm{fs}$ and $\tau_{s}=24.2 \mathrm{fs}$ ). The slow decay constant

corresponds to a more typical decay time of a Fabry-Pérot cavity. ${ }^{42-45}$ The two shorter decay times enhance the competition between radiative loss and photoinduced dynamics, and provide insights into these two directly competing and complementary deexcitation channels.

The photoemission $P_{\mathrm{em}}$ (cavity loss) and photodissociation probabilities $P_{\text {diss }}$ after excitation into the LP and UP mode are shown in Fig. 1. Comparing the dissociation probabilities after excitation into the LP, Fig. 1 a), c) and e), it becomes clear that the probability depends strongly on the cavity decay constant and less so on the number of molecules in the cavity. Here, the dissociation is in direct competition with cavity loss, so that the excitation of the system results in either dissociation or photon emission. The number of molecules only plays a noticeable role in these dynamics for e), the short-lived cavity, where an initial decay of the photonic contribution of the initial state within just a few fs takes place before the onset of nuclear dynamics. In this case, the photonic contribution of the initial state at early times depends on the number of molecules (see SI). The different photoemisson probability is not a consequence of a delay of the onset of photodissociation depending on the number of molecules, as seen by the equal arrival time of the 


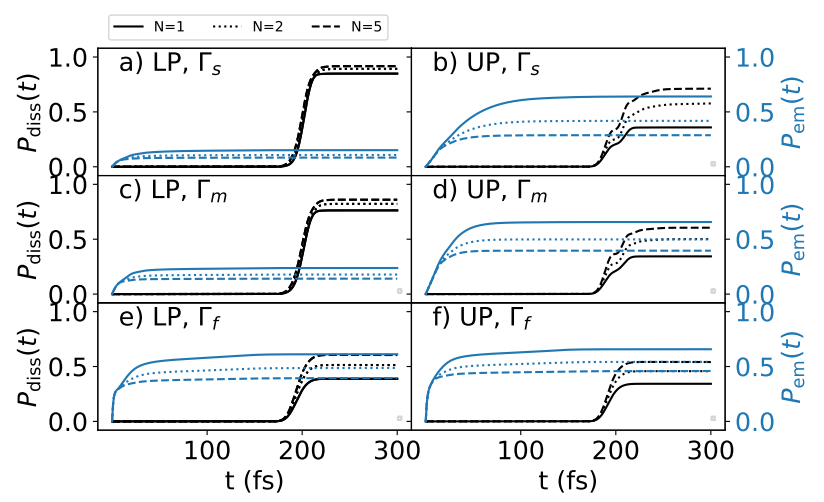

FIG. 1. Cumulative photodissociation $P_{\mathrm{diss}}(t)$ and photoemission probability $P_{\mathrm{em}}(t)$ after excitation into LP (left column) and UP mode (right column), for one, two, and five molecules $(N)$. The decay process of the cavity is slow $\left.\left.\left(\Gamma_{s}, \mathrm{a}\right)-\mathrm{b}\right)\right)$ to moderately fast $\left.\left.\left(\Gamma_{m}, \mathrm{c}\right)-\mathrm{d}\right)\right)$ to fast $\left.\left.\left(\Gamma_{f}, \mathrm{e}\right)-\mathrm{f}\right)\right)$.

wavepacket at the dissociation mark in Fig. 1e). This is contrast with an excitation to the UP (cf. Fig. 1b), 1d)) where the residence time at the FC region is clearly affected by the number of molecules. In a long-lived cavity, one of the molecules moves out of the FC region before the onset of the cavity decay. Thereafter, the decay channel is closed and this makes the probability of photodissociation, and in turn the probability of photoemission, mostly independent of the number of molecules. Indeed, in previous work we show how, for an infinitely long-lived cavity, the dissociation probability after LP excitation is practically independent of the number of molecules (for equal total coupling strength of the ensemble to the cavity) ${ }^{9}$ and this behaviour is recovered now for the slower cavities, Figs. 1 a) and c), albeit to a different extent. This behaviour is not recovered for the fast cavity, Fig. 1 e), due to the reduced photonic contribution of the cavity that cannot be replenished through Rabi cycling, as the cavity dynamics is on a different time scale (see SI).

An excitation into the UP state, Fig. 1 b), d) and f), leads to qualitatively and quantitatively very different dynamics. The number of molecules dominates the branching ratio between photodissociation and photoemission for all cavity lifetimes. A greater number of molecules leads to a higher dissociation yield and less photon leakage. This can be explained through the increasing density of dark states with increasing number of molecules: The effective UP lifetime decreases for an increased density of dark states, with the dark states being populated rapidly after excitation into the UP state. ${ }^{8}$ While the UP mode is populated, cavity decay can take place through the photonic 
contribution to the UP. With an increasing nonradiative decay rate towards the dark states, the radiative decay channel becomes less favorable and the net photon emission from the UP is reduced. The dark states however have no net contribution of the cavity mode, and therefore the radiative decay channel through the cavity is closed when the excitation resides in the dark states. Furthermore, dissociation can only occur from the LP mode and not the DSP, ${ }^{8,9}$ as the dark states are all bound states with respect to displacement along the nuclear coordinate. Finally, photodissociation occurs in the (nominally) LP state (cf. pPESs in Refs. ${ }^{8,9}$ ), which loses its photonic character as the chemical bond of the dissociating molecule elongates. This prevents the emission of a photon through the cavity, as only one of the two outcomes is possible - photodissociation or cavity decay.

One final and important aspect that is evident in Fig. 1 is that the dissociation dynamics of the molecule(s) for the short-lived (fast decay) cavity are identical irrespective of whether the initial population resides in the LP or the UP states. This implies that for this very short cavity lifetime, the polaritonic states are so short-lived that they do not contribute to the dynamics of the molecule and do not alter its "chemistry": The dissociation dynamics of the molecule in the short-lived cavity proceed in the same way as in the absence of a cavity.

The spectra after excitation of NaI into LP and UP states are shown in Figure 2. The LP peak appears broader than the UP peak due to the fast molecular dissociation from the LP state. ${ }^{9}$ For both the slow and moderately fast radiative decay, and one, two, and five molecules, the spectral features appear very similar. For the fast cavity decay, however (circle ticks), the LP and UP peaks are effectively smeared out and overlap substantially: LP and UP overlap and consequently a laser pulse would not be able to separately address them. This goes in line with the observations based on the dissociation dynamics: UP and LP states exhibit very similar dynamics and can no longer be viewed as separate states of the system. Conversely, when the LP and UP states are spectroscopically resolved, the dynamics emerging from each of the initial states differs, either in terms of the branching ratio between photodissociation and photoemission, or in terms of the delay time until the onset of photoemission, or both.

\section{B. Pyrazine: A multi-dimensional three-state system with internal conversion}

Pyrazine is a well-studied example of an N-heteroaromatic compound that undergoes rapid internal conversion (IC) from the $S_{2}$ to the $S_{1}$ electronic state upon photoexcitation ${ }^{65}$. This nonadiabatic process takes place on a time scale of a few tens of femtoseconds, similar to the time 


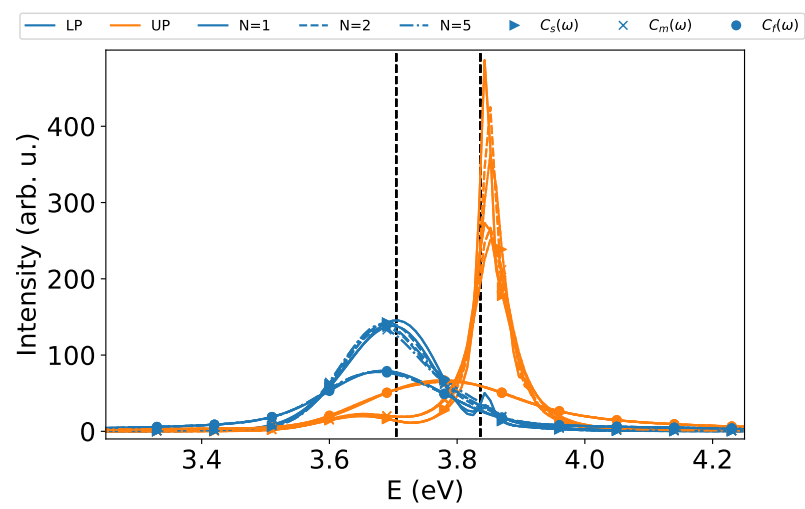

FIG. 2. Linear absorption spectra for an excitation into the pure polaritonic modes for $N$ NaI molecules and slow (s), moderately fast (m) and fast (f) cavity decay. The spectral position of the UP and LP mode is marked with black dashed lines. Symbols and lines in blue (orange) denote excitation into the LP (UP) mode.

scale of typical cavity decay times. Pyrazine, with its three electronic states, vibrational modes and nonadiabatic coupling represents an ideal exemplary molecule to investigate radiative decay and the role of polaritonic states in the photoinduced dynamics. Here, we consider only four vibrational modes explicitly, but note that models with all 24 vibrational modes have been formulated and used in quantum dynamics simulations. 56

\section{States $S_{0}-S_{2}$ resonant with the cavity mode}

In a first instance, the cavity resonance and coupling is chosen such that electronic states $\mathrm{S}_{0}$ and $\mathrm{S}_{2}$ are coupled. This is similar to NaI where now instead of photodissociation, the reactive channel is the IC process, and accumulated $\mathrm{S}_{1}$ population corresponds to product formation. Thus, cavity decay and IC ( $\mathrm{S}_{1}$ population) directly compete. The cavity-molecule interaction strength is chosen to result in a Rabi splitting of $\Omega_{R}=0.28 \mathrm{eV}$. As in the case of NaI, the Rabi splitting is kept constant with increasing number of molecules in the cavity through a scaling of the cavity-molecule coupling constant $g$ by $1 / \sqrt{N}$. This corresponds to a constant number density of molecules in the cavity independent of the ensemble size $N$.

By tuning the cavity photon energy it is possible to control which regions of configuration space are in resonance, thus differently affecting the system's dynamics. We consider here the resonance at the FC point, corresponding to a cavity frequency $\omega_{C}=4.72 \mathrm{eV}$. Resonance at the 


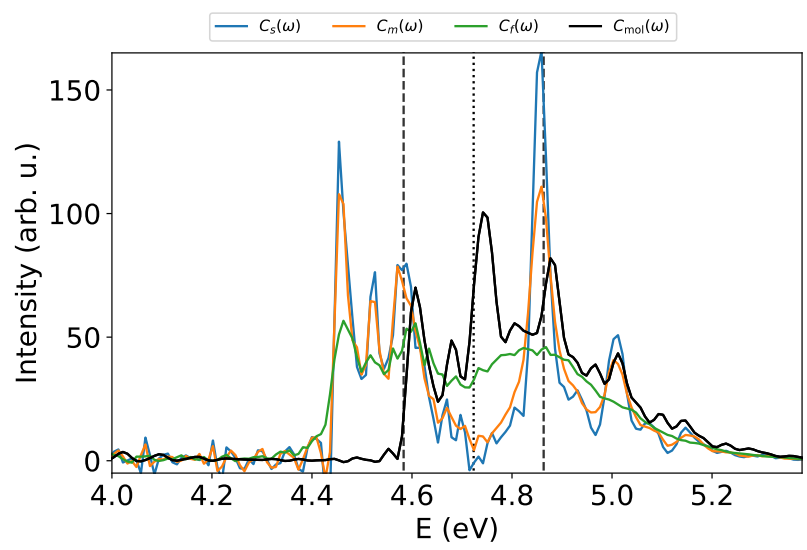

FIG. 3. Linear absorption for an excitation targeting the $S_{2}$ excited state; for $S_{0}-S_{2}$ coupled through the cavity and resonance at the FC point. One molecule in the cavity and also the bare molecule (in black) is shown. Three different cavity decay constants are selected, $\mathrm{f}$ (fast decay), m (moderately fast decay), and s (slow decay). The spectral position of the LP and UP states are marked with dashed vertical lines; the position of the $S_{2}$ energy at the FC point is marked with a black dotted line.

FC point maximizes collective effects due to the fact that all molecules have a large probability to be found around this geometry, whereas collective effects fade away quickly if the resonance condition involves other molecular geometries, as discussed with a simple model in the Supporting Information (SI).

The linear absorption spectrum (obtained through the Fourier transform of the autocorrelation function, $\left\langle\Psi(\mathbf{Q}, t=0 \mid \Psi(\mathbf{Q}, t\rangle\right.$ for a propagation of $120 \mathrm{fs})$ for vertical excitation from the $\mathrm{S}_{0}$ to the $\mathrm{S}_{2}$ electronic state through application of the dipole operator $\hat{T}_{S_{2}}$ is shown in Fig. 3 (for $N=1$ ). Overlaid in the Figure are spectra for three different decay constants, $\mathrm{f}$ (fast), $\mathrm{m}$ (moderately fast) and s (slow) corresponding to decay times of $\tau_{f}=4.84 \mathrm{fs}, \tau_{m}=24.2 \mathrm{fs}$ and $\tau_{s}=48.4 \mathrm{fs}$. First of all, the spectra of the molecule in the cavity are clearly distinguishable from the spectrum of the bare molecule (shown in black). Comparing the spectra for different decay constants, the moderately fast and slow decay constants lead to very similar spectra with well resolved LP and UP spectral regions. In contrast, the fast cavity decay leads to a broader spectrum without clearly defined polaritonic bands and closer to the spectrum of the free molecule.

To investigate the ensuing dynamics, a laser-pulse excitation of the molecule is carried out using two different laser pulses covering two different spectral regions, the low (pulse A) and high (pulse B) frequency region of the linear absorption spectrum, thus targeting LP and UP states, 

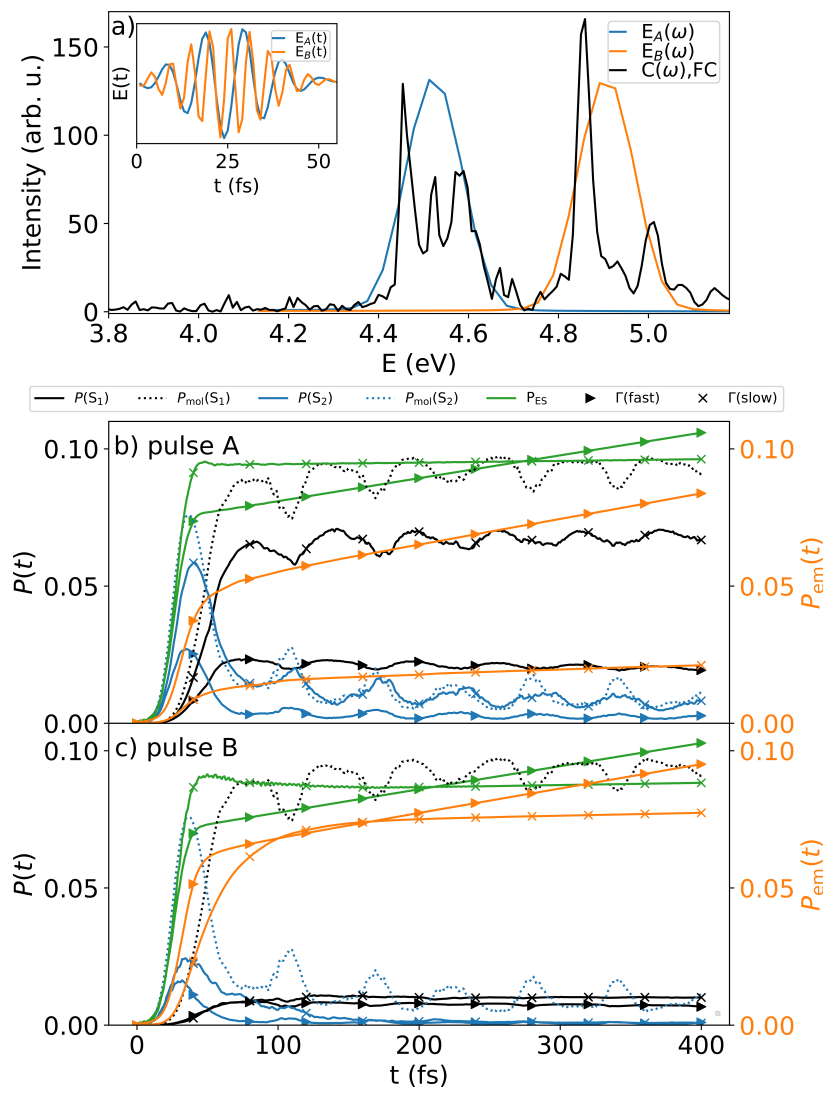

FIG. 4. a) Excitation laser pulse and spectrum for one molecule in the cavity and slow cavity decay, resonance at the FC point. b), c) System excitation probability $P_{\mathrm{ES}}(t)$, electronic excited state populations $P(t)$ and photoemission probability $P_{\mathrm{em}}(t)$ for laser pulse excitation into region A (lower energy, LP state) and region B (higher energy, UP state), for fast and slow cavity decay, resonance at the FC geometry and one molecule. The dynamics for the molecule without the cavity are also shown (dotted lines).

respectively. The laser pulses in frequency and time domain are shown below (Fig. 4 a)); and in the SI for the bare molecule excitation. To remain in the linear absorption regime, the laser intensity is chosen such that it yields an excitation probability of at most $10 \%$. Laser pulse parameters are given in the SI. For the simulations of molecular ensembles we do not use a dipolar-type excitation operator but directly excite the system with a laser pulse. This is technically simpler than applying an operator to a ML-MCTDH wavefunction (see SI) and leads to the same outcome concerning the dynamics of the excited states as long as the pulse intensity remains in the linear regime.

The electronic populations and photoemission probability triggered by pulses A and B are shown in Fig. 4 b) and c). Only the "fast" (f) and the "slow" (s) decay constants are shown, as the moderately fast cavity $(\mathrm{m})$ behaves similarly to (s). Comparing the dynamics upon excitation 
into the LP state, part b) of the Figure, for different decay times, the photoemission probability is clearly dominated by the decay constant. If the photoexcited system has not radiatively decayed in the first $120 \mathrm{fs}$ it remains trapped in $\mathrm{S}_{1}$. The intramolecular dynamics after excitation resemble the dynamics of the bare molecule, especially for the long-lived (slow) cavity; indicating that the LP state has strong $\mathrm{S}_{2}$ (molecular excited state) character. Noticeable is that the cavity continues to decay linearly for the short-lived case (large $\Gamma$ ). This continuation of the cavity decay is a result of strong coupling and the presence of virtual photons in the ensemble. In the description of the cavity decay terms introduced here, no distinction between real and virtual photons is made, and as a consequence, virtual photons can lead to cavity leakage as well, which of course is unphysical. This fact, however, is not relevant for our conclusions and could be numerically cured in the rotating wave approximation (RWA).

For an excitation into the UP state, Fig. 4c), the cavity decay time does not significantly affect the dynamics of the molecule. The single pyrazine molecule remains in the UP state because there are no dark states that result in a cavity-mediated nonradiative decay. ${ }^{8}$ Instead, from the UP state, almost all photoexcited population results in photoemission through the cavity and only a small fraction ends up in the $S_{1}$ electronic state via the natural nonradiative decay channel of the molecule. This indicates that the UP state exhibits stronger $\mathrm{S}_{0}$ (molecular ground state) character with a low IC probability, and a higher photoemission probability.

For the short lived (fast) cavity decay, LP (pulse A) and UP (pulse B) dynamics are quite similar and the intramolecular dynamics are not influenced substantially through interaction with the cavity. In this case, the corresponding spectrum in Fig. 3 does not resolve the LP and UP regions. As for $\mathrm{NaI}$ (for fast cavity decay), the polaritonic states are too short-lived to influence the dynamics of the system. Conversely, for the longer lived cavity, the laser pulses targeting the well resolved LP and UP spectral regions, as depicted in Fig. 4c), trigger different dynamical responses of the hybrid system.

We now turn to the collective effects on the relative photoemission probability $P_{\mathrm{em}}^{r}(t)$ by comparing one, two and five coupled molecules in the cavity. The relative photoemission probability shown in Fig. 5 is given as the fraction of photoemission probability over the total excitation probability by the laser pulse, $P_{\mathrm{ES}}^{\max }$, as $P_{\mathrm{em}}^{r}(t)=P_{\mathrm{em}}(t) / P_{\mathrm{ES}}^{\max }$. The total excitation probability is determined from the excitation of the corresponding ensemble with an infinite cavity lifetime. According to its definition, if all excitation were lost radiatively, $P_{\mathrm{em}}^{r}(t)$ would reach one in the long-time limit. Whenever the curves do not reach the theoretical maximum value of one, it is be- 


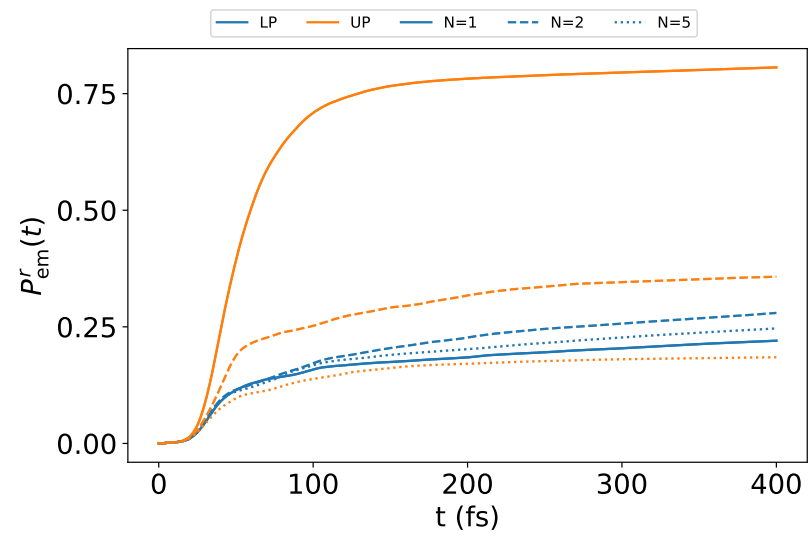

FIG. 5. Relative photoemission probability for pyrazine molecules in a cavity for the smallest decay constant $\Gamma_{s}$, normalized by the total excitation by the laser, $P_{\mathrm{ES}}^{\max }$. Shown are one, two and five molecules in the cavity and $\mathrm{S}_{0}-\mathrm{S}_{2}$ resonance at the FC point, with laser-pulse excitation targeting the LP and the UP mode.

cause the system has reached one of the $N$ nonradiative decay channels leading to intramolecular nonradiative decay of one of the $N$ pyrazine molecules into its $\mathrm{S}_{1}$ electronic state.

When the laser is tuned to the LP state, the probability of photoemission is virtually independent of the number of molecules. This is because the system can decay radiatively as long at it is found in the FC region, and as soon as one of the molecules leaves the FC region on the LP pPES, the photonic contribution of this state vanishes and no further photoemission is possible. It has been shown in the $\mathrm{NaI}$ example and previously ${ }^{9}$ that the time required for leaving the FC region of the LP pPES is independent of the number of molecules. Conversely, when the laser pulse populates the UP state, the photoemission probability becomes strongly dependent on the number of molecules. For only one molecule, the system spends a longer time in the UP state than for two and five molecules, and the photoemission probability is larger. As the number of molecules increases, the cavity-mediated internal conversion through collective conical intersections among dark states quickly depopulate the UP state towards the dark states manifold and subsequently towards the LP state, where the system can depart from the FC region. ${ }^{8}$ One sees that, already for five molecules, the photoemission probability after excitation onto the UP is smaller than for direct excitation into the LP state.

Summarizing, similar trends are found for the higher-dimensional pyrazine as are for NaI; as long as the polaritonic states appear as separate bands in the spectrum, the ensuing dynamics after excitation into either band are different, and are furthermore different from the dynamics of the 
free molecule. As such, the polaritonic states affect the chemistry of the system. If the cavity loss is so fast that the polaritonic states cannot be resolved spectroscopically, then the dynamics of the molecules in the cavity that retain the excitation resemble the dynamics of the free excited molecule.

\section{States $S_{1}-S_{2}$ resonant with the cavity mode}

We now investigate the case of a cavity resonant between $S_{1}$ and $S_{2}$. Albeit in pyrazine, the electronic transition between $S_{1}$ and $S_{2}$ is not dipole-allowed, we investigate this example to obtain further fundamental insights. As different resonance points on the PES, we select that the cavity is resonant at the $S_{1}$ minimum (vertical energy of $S_{2}$ ) and the $S_{2}$ minimum (vertical energy of $\mathrm{S}_{1}$ ). This results in cavity energies of $\omega_{C}=1.2603 \mathrm{eV}$ and $\omega_{C}=0.3657 \mathrm{eV}$, respectively. We again focus on the smallest decay constant $\Gamma_{S}$ in the following, and a single-molecule coupling $g \mu=0.13 \mathrm{eV}$. In this case, the cavity-molecule interaction strength is not scaled by $1 / \sqrt{N}$, for reasons that will become apparent below.

In this scenario, the intramolecular nonadiabatic coupling and the cavity mode couple the same molecular states, namely $S_{1}$ and $S_{2}$, and thus the cavity opens a new channel for IC towards $S_{1}$. The population of $S_{1}$ alone does not signify which channel is dominant, but a comparison of the photoemission probability, and $S_{2}$ and $S_{1}$ population.

In Fig. 6, pPES cuts from a diagonalization of the Tavis-Cummings Hamiltonian are shown for the two selected resonances a) at the $S_{1}$ minimum and $b$ ) at the $S_{2}$ minimum. It should be noted that these potentials are not used in the dynamics, they merely serve as a device to discuss and explain the dynamical evolution of the system. ${ }^{9}$ Overlaid in the Figure are the uncoupled $\mathrm{S}_{1}$ and $\mathrm{S}_{2}$ PESs, with the $\mathrm{S}_{1}$ PES shifted by the respective photon energy of the cavity. Comparing the two different scenarios a) and b), the (nominal) LP and UP pPES follow the uncoupled PESs closely, except in the vicinity of the resonance. Away from the resonance, LP and UP states are identical to either the $S_{1}$ or $S_{2}$ electronic states.

In Fig. 7, the electronic populations and photoemission probabilities are shown after excitation into the $\mathrm{S}_{2}$ electronic state using the same laser pulse as for the bare molecule, for one, two and five molecules. Fig. 7a) illustrates the evolution of the system when the cavity is resonant with the energy difference between $S_{1}$ and $S_{2}$ at the $S_{1}$ minimum geometry. The $S_{1}$ PES dressed by the photon energy of the cavity lies close to the $\mathrm{S}_{2}$ PES at the FC point. The wave packet leaves this 

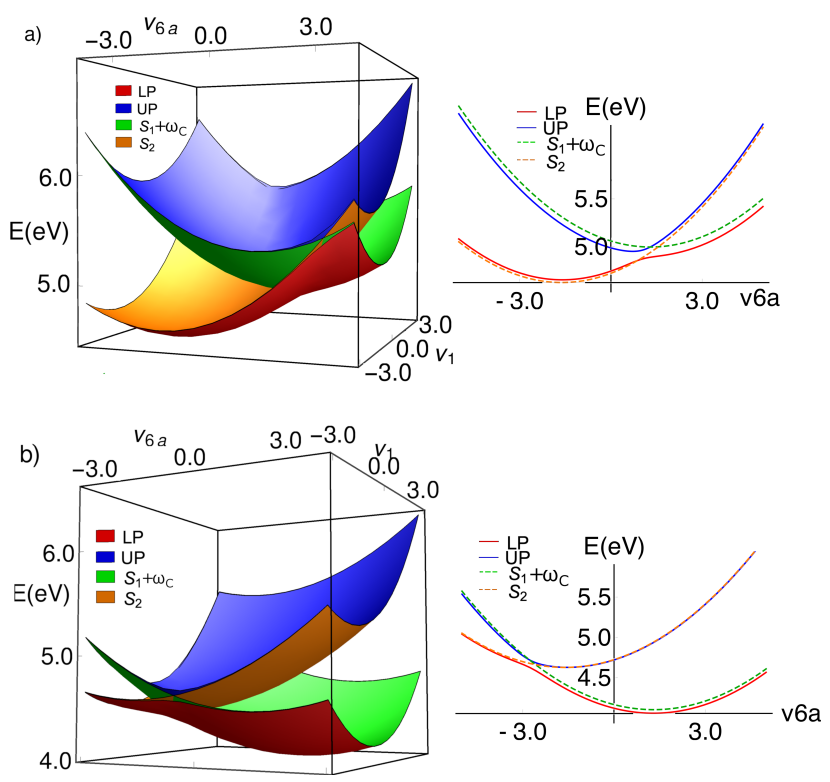

FIG. 6. PES cuts for pyrazine and $S_{1}-S_{2}$ coupling with resonance a) at $S_{1, \min }$; b) at $S_{2, \min }$. Blue and red surfaces depict LP and UP for one molecule in the cavity, obtained through diagonalization of the TavisCummings Hamiltonian. The molecule is displaced along modes $v_{6 a}$ and $v_{1}$ with $v_{10 a}$ and $v_{9 a}$ held fixed (to their respective values at the $S_{2}$ and $S_{1}$ minimum for the 2D surface cuts, and to zero (the Franck-Condon point) for the 1D surface cuts). Green and orange surfaces depict the PESs for uncoupled electronic states $S_{1}$ shifted by the respective cavity photon energy $\omega_{C}$ and $S_{2}$. While in a), the LP mostly carries the character of $S_{2}$ and the UP of $S_{1}$, the situation is reversed in $b$ ).

region quickly after photoexcitation and hence the time evolution is very similar to the dynamics of the free molecule, such that almost no cavity decay takes place. Fig. 7b) illustrates the time evolution when the cavity is resonant at the $S_{2}$ minimum. After excitation to $S_{2}$, the wave packet quickly evolves towards the $S_{2}$ minimum via a downhill pathway since the PES of $S_{2}$ at the FC point lies energetically well above the configuration at which the cavity couples states $S_{1}$ and $\mathrm{S}_{2}$, as seen in the inset in Fig. 7b). The dynamics after excitation are then quite different from the dynamics of the free molecule: The final state $S_{1}$ is also reached through the radiative decay channel (through the cavity), by about 50\%, apparent from the photoemission probability (orange curve). In this case, the radiative decay channel competes with the nonradiative (nonadiabatic) decay channel from $S_{2}$ to $S_{1}$, and the nonadiabatic decay channel is no longer the only channel taken to the product state $S_{1}$.

This example illustrates how the dynamics can be influenced by the choice of the cavity reso- 


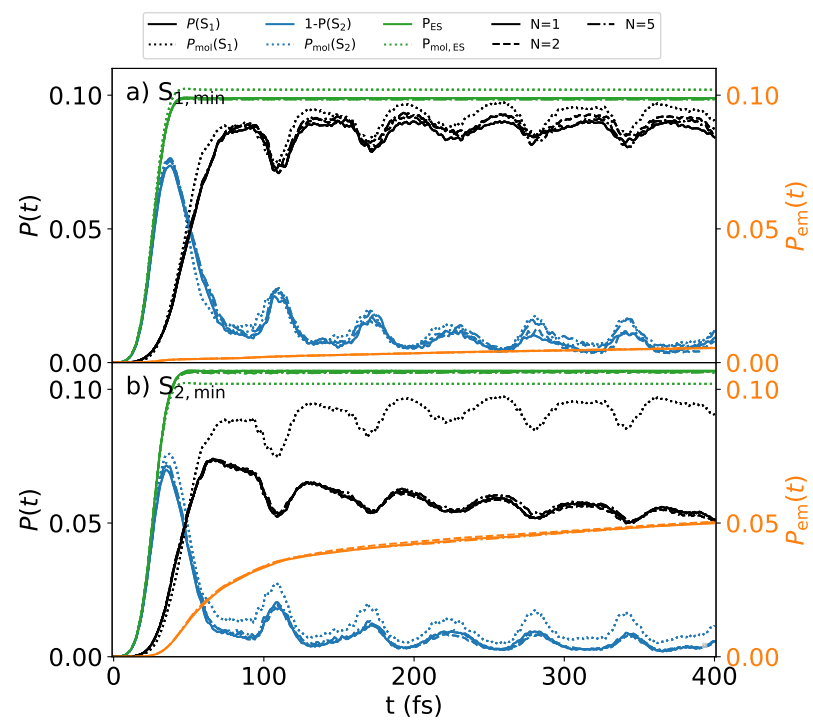

FIG. 7. Populations of electronic states and decay after excitation into $S_{2}$ using a laser pulse, for one, two and five molecules. a) Resonance at the $S_{2, \min }$; b) resonance at the $S_{1, \min }$. In a), the electronic populations behave similar to the free molecule with little cavity decay, whereas in b) cavity decay is observed and the IC process is suppressed compared to the bare molecule.

nance. Generally, a substantial cavity mode population and subsequent relaxation can be expected if the cavity is resonant with the minimum energy geometry of the initially populated electronic state, in this case $S_{2}$. The system reaches this geometry through an energetically favorable pathway. For pyrazine, in particular, the molecules can still reach the region of pristine nonadiabatic coupling and a competition between both processes takes place. In the case that the cavity is resonant at the minimum of the final state, in this case $S_{1}$, it can occur, as for pyrazine, that the wave packet cannot come close to such geometries, which lie high in energy in the $S_{2}$ PES, and the dynamics proceeds as if no cavity would be present. It should be possible to verify these kinds of observations through time-resolved spectroscopic measurements in cavities of different frequencies.

Finally, we note in passing that in this coupling case there are no collective effects for $N>1$ in the single-excitation manifold (absorption of one photon): This is clear from the identical curves for $N=2$ and $N=5$ in Fig. 7. In the single-excitaton manifold, one molecule undergoes the photoinitiated dynamics and cavity interaction involving $S_{2}$ and $S_{1}$, while all other molecules reside in $\mathrm{S}_{0}$. Only for an excitation of several molecules at once, collective effects similar to those in the $\mathrm{S}_{0}-\mathrm{S}_{n}$ coupling case, can appear again, which should be investigated separately. 


\section{States $S_{0}-S_{1}$ resonant with the cavity mode}

Finally, we investigate the situation in which the electronic state after nonradiative decay, $\mathrm{S}_{1}$, is coupled to the ground electronic state $S_{0}$ via the cavity. Such a resonance is interesting in terms of photonic applications and transport properties, as well as energy conversion - a broadband excitation into the $S_{2}$ state is followed by IC and increases population of the $S_{1}$ electronic state. Through the subsequent population of the cavity-matter hybrid states, an emission of a photon with a defined wavelength can be achieved. In this work we only consider the single-photon manifold (no simultaneous excitation of two or more molecules) and remind that, in this manifold, and because $\mathrm{S}_{0}$ is involved, collective effects are possible. As before, different resonance points on the molecular PES can be chosen, and we consider resonance at the FC point (vertical $\mathrm{S}_{1}$ energy), at the $S_{1}$ minimum (vertical energy of $S_{0}$ ) and the minimum of the CI seam (vertical energy of $S_{1}$ and $S_{0}$ ). We focus here on the cavity with the smallest decay constant, $\Gamma_{s}$ (decay time of $48 \mathrm{fs}$ ). The Rabi splitting is adjusted to $\Omega_{R}=0.13 \mathrm{eV}$ for one molecule and not scaled with $1 / \sqrt{N}$ in the following. The cavity frequencies amount to $\omega_{C}=3.81 \mathrm{eV}(\mathrm{FC}), \omega_{C}=3.495 \mathrm{eV}\left(\mathrm{S}_{1}\right.$ minimum$)$, and $\omega_{C}=3.97 \mathrm{eV}(\mathrm{CI}$ minimum). The laser pulse that is used for the initial excitation of the molecules is the same pulse as for the free molecule (see SI).

In Figure 8, the electronic populations, excitation probability, and photoemission probability are shown for one to seven molecules in a cavity resonant at the FC point, $\mathrm{S}_{1}$ minimum and minimum of the CI seam, for the long-lived cavity.

Collective effects are possible in these cases, and they would manifest as ensemble-size dependent state populations and photoemission probabilities. As the cavity-molecule coupling per molecule (and not the overall Rabi splitting) is kept constant, any deviation of the radiative and nonradiative decay characteristics for larger ensemble sizes directly indicates the presence of collective effects. However, the molecule of the ensemble that absorbs the photon and is excited to $\mathrm{S}_{2}$ and those that remain in their ground electronic and vibrational state consist of potentially very different probability densities along their vibrational coordinates (cf. Eqs. $(2,3)$ in the SI). As the numerical simulations and simple model considerations introduced in the SI illustrate, this leads to collective effects that scale weakly with the number of molecules and involve, at most, a handful of molecules simultaneously.

In all cases, the initial coherent excitation of the ensemble proceeds in the same way and corresponds to the green curves in Fig. 8. Initially, $\mathrm{S}_{2}$ (blue curves) becomes populated and imme- 


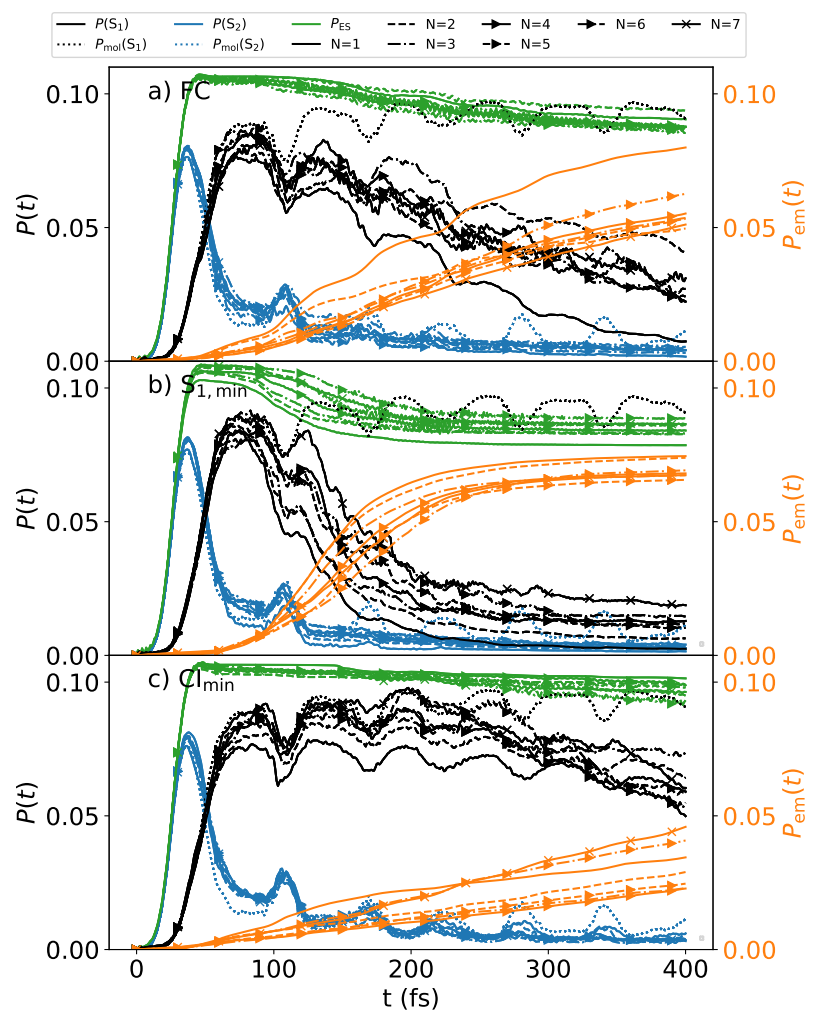

FIG. 8. Populations of the electronic states and decay after laser-pulse excitation into $S_{2}$ for one to seven molecules and slow cavity decay. The cavity is resonant with $S_{0}$ and $S_{1}$ at a) the FC point; b) the $S_{1, \min }$; c) the $\mathrm{CI}_{\min }$. Here, the cavity-molecule interaction strength is not scaled with the number of molecules, so that the coupling per molecule is identical for increasing $N$, leading to an increased Rabi splitting of the pPES for configurations in which more than one molecule is resonant with the cavity at a time.

diately thereafter population starts to be transferred to $S_{1}$ (black curves) via the pristine conical intersection. As $S_{1}$ becomes populated, the cavity mode starts to participate in the dynamics and the cavity can decay, emitting a photon (orange curves). The sum of the $S_{2}, S_{1}$ population and photoemission probability adds up to the total excitation probability. The branching between $S_{1}$ population and photoemission depends strongly on the location of the resonance, with the populations for the three locations shown in Figs. 8a), b) and c). In all of the examples, a larger number of molecules leads to a reduced photoemission and a consequently larger probability the system remains in $S_{1}$. The effect is most pronounced for resonance at the Franck-Condon point, where the molecules besides the photoexcited one can participate in the dynamics involving the cavity mode. Nonetheless, collective effects extend only marginally beyond $N=2$ molecules.

For a resonance at the $\mathrm{FC}$ point, the molecules that are not photoexcited can participate in the 
collective dynamics once the photoexcited molecule reaches this geometry again via $\mathrm{S}_{1}$. Recall that all molecules have equal probability to be photoexcited but only one of them is actually promoted to $S_{2}$ by the laser pulse in the single excitation regime. Once the photoexcited molecule reaches the resonant FC geometry in its pathway along the $S_{1}$ PES, it can interact with the cavity and transfer to $\mathrm{S}_{0}$ while the cavity becomes excited. The photoexcited cavity can now either decay, or transfer its excitation to any of the molecules, since all are resonant with the $S_{0}-S_{1}$ transition. An immediate consequence of this reasoning is that the shorter-lived the cavity, the less collective dynamics can be expected, as the photon will be released before initially non-excited molecules can become excited via the cavity.

In the case of cavity resonance at the $S_{1}$ minimum, the situation is reversed: The excited molecule will reach the region of the $S_{1}$ minimum and interact with the cavity. The unexcited molecules however can only partake in the collective dynamics in case their nuclear probability density is not negligible at this resonance geometry. A detailed explanation of this phenomenon based on simple model assumptions is provided in the SI. A similar analysis has been carried out in Ref. [66], although limited to two molecules in the cavity. In this example, the cavitymolecule resonance only occurs later in the dynamics but not at the initial, stationary point, and thus collective effects are very weak.

This analysis concludes that the probability that more than a handful of the unexcited molecules are found simultaneously at a geometry different from their equilibrium position becomes quickly negligible as more molecules are considered, and as a consequence, collective effects are weak, as readily seen by following by eye the various orange curves.

Finally, in the case of resonance at the CI minimum, the excited molecule cannot efficiently reach the region of resonance with the cavity and the molecules in the ground electronic state have a small probability density in this region. Both effects lead to the smallest photoemission and the largest probability that the photoexcited molecule remains in $S_{1}$.

Summarizing, if only one molecule would interact with the cavity at a given time, then the dynamics of the ensemble would be independent of the number of molecules. We see that some involvement of other molecules takes place, but it can be hindered by both the cavity decay and the low probability density to find the unexcited molecules at other geometries different than the FC geometry.

To further illustrate the participation of molecules that had not been initially excited by the laser, and to discuss the possible role of coherences resulting from the coherent excitation of the 


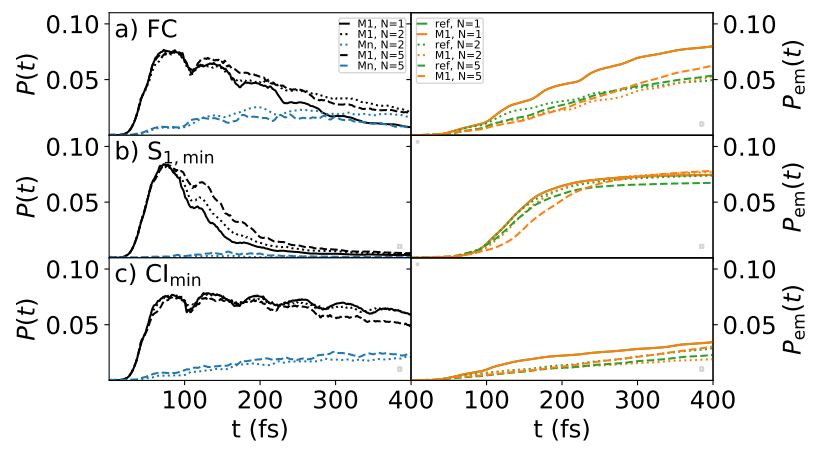

FIG. 9. $\mathrm{S}_{1}$ excited state populations $P(t)$ of the molecule coupled to the laser pulse (M1) and sum over all other molecules $(\mathrm{Mn})$; further, photoemission probability $P_{\mathrm{em}}(t)$ of the cavity where only one molecule is coupled to the laser pulse (M1) and for the reference cavity, where all molecules are coupled to the laser pulse (ref). Shown are one, two and five molecules in the cavity coupling states $S_{0}$ and $S_{1}$ for an interaction strength that is not scaled with the number of molecules in the cavity. The resonant points of the potential are a) the FC point, b) the $S_{1}$ potential minimum, c) the potential minimum along the CI seam.

ensemble, a second set of simulations with the exact same cavity parameters, but only one of the molecules coupled to the (unscaled) laser pulse has been carried out and the results are shown in Fig. 9. In this scenario, strictly just one molecule (M1) is excited to the $\mathrm{S}_{2}$ state and undergoes IC. Note that, in the previous discussion, also only one molecule was excited at a time, but the wave function consisted of a coherent superposition including a term for each possibly photoexcited molecule, each with a weight of $1 / \sqrt{N}$, as shown in Eq. (2) of the SI. The $\mathrm{S}_{1}$ population of the excited molecule is shown in Fig. 9 as black curves, and the $S_{1}$ population of the initially unexcited molecules is shown as blue curves. If no collective effects were present, this latter population would remain equal to zero throughout. The fact that molecules that were initially not excited can be excited via the cavity is the reason for the smaller emission probability as the number of molecules increases. As we have already discussed though, this effect scales poorly with the number of molecules.

The photoemission probability of the cavity with only one molecule coupled to the laser pulse is shown on the right-hand side of Fig. 9 (M1), together with the reference probability for all molecules coupled to the laser pulse (ref). The overall photoemission probability curves over time are very similar for the two cases, coherent and incoherent single-molecule excitation, demonstrating that interference effects do not play a role. 


\section{CONCLUSION}

The role of the cavity lifetime on the ultrafast dynamics following the photoexcitation of a single molecule and molecular ensembles has been investigated by real-time quantum wave packet propagations. We demonstrate that, as long as the LP and UP bands can be resolved spectroscopically (their linewidths are narrow enough that the Rabi splitting is visible), the excitation of the system into either the LP or UP states leads to a different collective response and to modified and differentiated photophysics and photochemistry. If, however, the radiative cavity decay is so fast that the LP and UP bands are not resolved in the spectrum, then the dynamics of the molecule or the ensemble are also not impacted by the cavity. This fact is linked with the definition of the weak and strong coupling regimes, where a sole energetic criterion to classify "weak" and "strong" coupling is not sufficient, as has been discussed previously in the experimental literature. ${ }^{49,50}$

For a cavity resonant with the $S_{2}-S_{1}$ transition of a molecule (i.e. not involving $S_{0}$ ) collective effects are strictly not possible in the single excitation manifold. Nonetheless, the choice of the resonance location of the cavity provides a handle to control the branching between nonradiative decay and photoemission via coupling to the cavity mode. In particular, if the cavity is made to be resonant at the minimum energy configuration of the electronic state reached by photoexcitation, the wave packet can favorably reach this point and the coupling to the cavity mode and the subsequent photoemission become the dominating channel.

In the case that the cavity couples the $S_{1}-S_{0}$ electronic states of the molecules in the ensemble, collective effects are possible because the ground electronic state is involved. We show, however, that in this case collective effects are rather weak, specially when the cavity is resonant away from the FC point of the unexcited molecules. The collective effects converge rapidly with an increasing number of molecules as the resonant phase-space volume remains small as compared to the available phase-space. Collective effects might become more important in the multi-photon regime when more than one molecule is excited simultaneously by the laser pulse and subsequently reach the configuration resonant with the cavity mode simultaneously. Such kinds of effects remain to be studied in future work. 


\section{CREDIT LINE}

The following article has been accepted at The Journal of Chemical Physics. After it is published, it will be found at Link.

\section{SUPPORTING INFORMATION}

See Supporting Information for computational details of the calculations; a note concerning Rabi oscillations and the decay terms; details about the photonic contribution in the NaI-cavity ensemble; the leakage of photons through loss of virtual photons for the higher coupling strength; the laser pulses and laser pulse parameters for excitation targeting $\mathrm{S}_{2}$, LP and UP; single-molecule coupling $\mathrm{S}_{1}$ population and decay characteristics; and a model discussion of the collective effects in the $\mathrm{S}_{1}-\mathrm{S}_{0}$ coupling case.

\section{DATA AVAILABILITY STATEMENT}

The data that support the findings of this study are available from the corresponding author upon reasonable request.

\section{REFERENCES}

${ }^{1}$ D. Sanvitto and S. Kéna-Cohen, “The Road towards Polaritonic Devices,” Nat. Mater. 15, 10611073 (2016).

${ }^{2}$ T. W. Ebbesen, "Hybrid Light-Matter States in a Molecular and Material Science Perspective," Acc. Chem. Res. 49, 2403-2412 (2016).

${ }^{3}$ D. G. Baranov, M. Wersäll, J. Cuadra, T. J. Antosiewicz, and T. Shegai, "Novel Nanostructures and Materials for Strong Light-Matter Interactions," ACS Photonics 5, 24-42 (2018).

${ }^{4}$ M. Hertzog, M. Wang, J. Mony, and K. Börjesson, "Strong Light-Matter Interactions: A New Direction within Chemistry," Chem. Soc. Rev. 48, 937 (2019).

${ }^{5}$ A. Thomas, L. Lethuillier-Karl, K. Nagarajan, R. M. Vergauwe, J. George, T. Chervy, A. Shalabney, E. Devaux, C. Genet, J. Moran, and T. W. Ebbesen, "Tilting a ground-state reactivity landscape by vibrational strong coupling," Science 363, 615-619 (2019). 
${ }^{6}$ V. M. Agranovich, Y. N. Gartstein, and M. Litinskaya, "Hybrid Resonant Organic-Inorganic Nanostructures for Optoelectronic Applications," Chem. Rev. 111, 5179-5214 (2011).

${ }^{7}$ J. Feist and F. J. Garcia-Vidal, "Extraordinary Exciton Conductance Induced by Strong Coupling,” Phys. Rev. Lett. 114, 1-5 (2015).

${ }^{8}$ O. Vendrell, "Collective Jahn-Teller Interactions through Light-Matter Coupling in a Cavity," Phys. Rev. Lett. 121, 253001 (2018).

${ }^{9}$ I. S. Ulusoy, J. A. Gomez, and O. Vendrell, "Modifying the nonradiative decay dynamics through conical intersections via collective coupling to a cavity mode," J. Phys. Chem. A 123, 8832-8844 (2019).

${ }^{10}$ E. Orgiu, J. George, J. A. Hutchison, E. Devaux, J. F. Dayen, B. Doudin, F. Stellacci, C. Genet, J. Schachenmayer, C. Genes, G. Pupillo, P. Samorì, and T. W. Ebbesen, "Conductivity in Organic Semiconductors Hybridized with the Vacuum Field," Nat. Mater. 14, 1123-1129 (2015).

${ }^{11}$ X. Zhong, T. Chervy, S. Wang, J. George, A. Thomas, J. A. Hutchison, E. Devaux, C. Genet, and T. W. Ebbesen, “Non-Radiative Energy Transfer Mediated by Hybrid Light-Matter States," Angew. Chemie - Int. Ed. 55, 6202-6206 (2016).

${ }^{12}$ M. Du, L. A. Martínez-Martínez, R. F. Ribeiro, Z. Hu, V. M. Menon, and J. Yuen-Zhou, “Theory for Polariton-Assisted Remote Energy Transfer,” Chem. Sci. 9, 6659-6669 (2018).

${ }^{13}$ C. Schäfer, M. Ruggenthaler, H. Appel, and A. Rubio, "Modification of Excitation and Charge Transfer in Cavity Quantum-Electrodynamical Chemistry," PNAS 116, 4883-4892 (2019).

${ }^{14}$ E. Eizner, J. Brodeur, F. Barachati, A. Sridharan, and S. Kéna-Cohen, “Organic Photodiodes with an Extended Responsivity Using Ultrastrong Light-Matter Coupling," ACS Photonics 5, 2921-2927 (2018).

${ }^{15}$ M. Litinskaya and F. Herrera, "Vacuum-Enhanced Optical Nonlinearities with Disordered Molecular Photoswitches,” Phys. Rev. B 99, 041107 (2019).

${ }^{16}$ A. Graf, L. Tropf, Y. Zakharko, J. Zaumseil, and M. C. Gather, "Near-Infrared ExcitonPolaritons in Strongly Coupled Single-Walled Carbon Nanotube Microcavities,” Nat. Commun. 7, 13078 (2016).

${ }^{17}$ A. Graf, M. Held, Y. Zakharko, L. Tropf, M. C. Gather, and J. Zaumseil, "Electrical Pumping and Tuning of Exciton-Polaritons in Carbon Nanotube Microcavities," Nat. Mater. 16, 911 (2017).

${ }^{18}$ E. Jaynes and F. Cummings, "Comparison of Quantum and Semiclassical Radiation Theories with Application to the Beam Maser,” Proc. IEEE 5, 89-109 (1963). 
${ }^{19}$ M. Tavis and F. W. Cummings, “Approximate Solutions for an N-Molecule-Radiation-Field Hamiltonian,” Phys. Rev. 188, 692-695 (1969).

${ }^{20}$ J. Feist, J. Galego, and F. J. Garcia-Vidal, "Polaritonic Chemistry with Organic Molecules," ACS Photonics 5, 205-216 (2018).

${ }^{21}$ T. Szidarovszky, G. J. Halasz, A. G. Csaszar, and L. S. Cederbaum, “Conical Intersections Induced by Quantum Light: Field-Dressed Spectra from the Weak to the Ultrastrong Coupling Regimes,” J. Phys. Chem. Lett. 9, 6215-6223 (2018).

${ }^{22}$ M. Ruggenthaler, J. Flick, C. Pellegrini, H. Appel, I. V. Tokatly, and A. Rubio, "QuantumElectrodynamical Density-Functional Theory: Bridging Quantum Optics and ElectronicStructure Theory," Phys. Rev. A - At. Mol. Opt. Phys. 90, 1-26 (2014).

${ }^{23}$ J. Flick, C. Schäfer, M. Ruggenthaler, H. Appel, and A. Rubio, “Ab Initio Optimized Effective Potentials for Real Molecules in Optical Cavities: Photon Contributions to the Molecular Ground State," ACS Photonics 5, 992-1005 (2018).

${ }^{24}$ N. M. Hoffmann, H. Appel, A. Rubio, and N. T. Maitra, "Light-Matter Interactions via the Exact fFactorization Approach,” Eur. Phys. J. B 91, 180 (2018).

${ }^{25}$ A. Abedi, E. Khosravi, and I. V. Tokatly, "Shedding Light on Correlated Electron-Photon States using the Exact Factorization,” Eur. Phys. J. B 91, 194 (2018).

${ }^{26}$ L. Lacombe, N. M. Hoffmann, and N. T. Maitra, "Exact Potential Energy Surface for Molecules in Cavities," Phys. Rev. Lett. 123, 083201 (2019).

${ }^{27}$ O. Vendrell, "Coherent Dynamics in Cavity Femtochemistry: Application of the MultiConfiguration Time-Dependent Hartree Method," Chem. Phys. 509, 55-65 (2018).

${ }^{28}$ A. Csehi, G. J. Halász, and Á. Vibók, "Collective Effect of Light-Induced and Natural Nonadiabatic Phenomena in the Dissociation Dynamics of the NaI Molecule," Chem. Phys. 509, 91-97 (2018).

${ }^{29}$ J. F. Triana and J. L. Sanz-Vicario, "Revealing the Presence of Potential Crossings in Diatomics Induced by Quantum Cavity Radiation,” Phys. Rev. Lett. 122, 063603 (2019).

${ }^{30}$ T. Neuman and J. Aizpurua, "Origin of the Asymmetric Light Emission from Molecular ExcitonPolaritons," Optica 5, 1247 (2018).

${ }^{31}$ A. Strashko, P. Kirton, and J. Keeling, "Organic Polariton Lasing and the Weak to Strong Coupling Crossover,” Phys. Rev. Lett. 121, 193601 (2018).

${ }^{32}$ L. A. Martínez-Martínez, E. Eizner, S. Kéna-Cohen, and J. Yuen-Zhou, “Triplet Harvesting in the Polaritonic Regime: A Variational Polaron Approach,” J. Chem. Phys 151, 54106 (2019). 
${ }^{33}$ H. L. Luk, J. Feist, J. J. Toppari, and G. Groenhof, "Multiscale Molecular Dynamics Simulations of Polaritonic Chemistry,' J. Chem. Theory Comput. 13, 4324-4335 (2017).

${ }^{34}$ G. Groenhof, U. Climent, J. Feist, D. Morozov, and J. Jussi Toppari, “Tracking Polariton Relaxation with Multiscale Molecular Dynamics Simulations,” J. Phys. Chem. Lett. 10, 5476-5483 (2019).

${ }^{35}$ F. Herrera and F. C. Spano, "Dark Vibronic Polaritons and the Spectroscopy of Organic Microcavities,” Phys. Rev. Lett. 118, 223601 (2017).

${ }^{36}$ F. Herrera and F. C. Spano, “Absorption and Photoluminescence in Organic Cavity QED,” Phys. Rev. A 95, 1-25 (2017).

${ }^{37}$ F. Herrera and F. C. Spano, "Theory of Nanoscale Organic Cavities: The Essential Role of Vibration-Photon Dressed States," ACS Photonics 5, 65-79 (2018).

${ }^{38}$ D. G. Lidzey, A. M. Fox, M. D. Rahn, M. S. Skolnick, V. M. Agranovich, and S. Walker, "Experimental study of light emission from strongly coupled organic semiconductor microcavities following nonresonant laser excitation,” Phys. Rev. B 65, 195312 (2002).

${ }^{39}$ V. M. Agranovich, M. Litinskaia, and D. G. Lidzey, “Cavity Polaritons in Microcavities Containing Disordered Organic Semiconductors,” Phys. Rev. B - Condens. Matter Mater. Phys. 67, $1-10$ (2003).

${ }^{40}$ M. Litinskaya, P. Reineker, and V. M. Agranovich, "Fast Polariton Relaxation in Strongly Coupled Organic Microcavities," J. Lumin. 110, 364-372 (2004).

${ }^{41}$ T. Virgili, D. Coles, A. M. Adawi, C. Clark, P. Michetti, S. K. Rajendran, D. Brida, D. Polli, G. Cerullo, and D. G. Lidzey, "Ultrafast Polariton Relaxation Dynamics in an Organic Semiconductor Microcavity," Phys. Rev. B 83, 245309 (2011).

${ }^{42}$ T. Schwartz, J. A. Hutchison, J. Léonard, C. Genet, S. Haacke, and T. W. Ebbesen, "Polariton Dynamics under Strong Light-Molecule Coupling," Chem. Phys. Chem. 14, 125-131 (2013).

${ }^{43}$ S. Wang, T. Chervy, J. George, J. A. Hutchison, C. Genet, and T. W. Ebbesen, "Quantum Yield of Polariton Emission from Hybrid Light-Matter States,” J. Phys. Chem. Lett. 5, 1433-1439 (2014).

${ }^{44}$ J. George, S. Wang, T. Chervy, A. Canaguier-Durand, G. Schaeffer, J.-M. Lehn, J. A. Hutchison, C. Genet, and T. W. Ebbesen, "Ultra-Strong Coupling of Molecular Materials: Spectroscopy and Dynamics," Faraday Discuss. 178, 281 (2015).

${ }^{45}$ J. Mony, M. Hertzog, K. Kushwaha, and K. Börjesson, “Angle-Independent Polariton Emission Lifetime Shown by Perylene Hybridized to the Vacuum Field Inside a Fabry-Pérot Cavity," J. 
Phys. Chem. C 122, 24917-24923 (2018).

${ }^{46}$ A. Canaguier-Durand, C. Genet, A. Lambrecht, T. W. Ebbesen, and S. Reynaud, "NonMarkovian Polariton Dynamics in Organic Strong Coupling,” Eur. Phys. J. D 69, 24 (2015).

${ }^{47}$ B. Xiang, R. F. Ribeiro, L. Chen, J. Wang, M. Du, J. Yuen-Zhou, and W. Xiong, "State-Selective Polariton to Dark State Relaxation Dynamics," J. Phys. Chem. A 123, 5918 (2019).

${ }^{48}$ P. Törmö and W. L. Barnes, "Strong Coupling Between Surface Plasmon Polaritons and Emitters: A Review,” Reports Prog. Phys. 78, 013901 (2015), arXiv:1405.1661v1.

${ }^{49}$ R. Thomas, A. Thomas, S. Pullanchery, L. Joseph, S. M. Somasundaran, R. S. Swathi, S. K. Gray, and K. G. Thomas, "Plexcitons: The Role of Oscillator Strengths and Spectral Widths in Determining Strong Coupling Article,” ACS Nano 12, 402-415 (2018).

${ }^{50}$ H. Wang, H. Y. Wang, H. B. Sun, A. Cerea, A. Toma, F. De Angelis, X. Jin, L. Razzari, D. Cojoc, D. Catone, F. Huang, and R. Proietti Zaccaria, "Dynamics of Strongly Coupled Hybrid States by Transient Absorption Spectroscopy," Adv. Funct. Mater. 28, 1-22 (2018).

${ }^{51}$ H. D. Meyer, U. Manthe, and L. S. Cederbaum, "The Multi-Configurational Time-Dependent Hartree Approach,” Chem. Phys. Lett. 165, 73-78 (1990).

${ }^{52}$ M. H. Beck, A. Jäckle, G. A. Worth, and H.-D. Meyer, "The Multiconfiguration TimeDependent Hartree (MCTDH) Method: A Highly Efficient Algorithm for Propagating Wavepackets," Phys. Rep. 324, 1-105 (2000).

${ }^{53}$ L. S. Cederbaum and F. Tarantelli, "Nuclear Dynamics of Decaying States: A Time-Dependent Formulation,” J. Chem. Phys. 98, 9691-9706 (1993).

${ }^{54}$ N. Moiseyev, "Quantum Theory of Resonances: Calculating Energies, Widths and CrossSections by Complex Scaling," Phys. Rep. 302, 211-293 (1998).

${ }^{55}$ G. A. Worth, H. Meyer, and L. S. Cederbaum, "The Effect of a Model Environment on the $\mathrm{S}_{2}$ Absorption Spectrum of Pyrazine: A Wave Packet Study Treating all 24 Vibrational Modes," J. Chem. Phys. 105, 4412-4426 (1996).

${ }^{56}$ A. Raab, G. A. Worth, H.-D. Meyer, and L. S. Cederbaum, "Molecular Dynamics of Pyrazine after Excitation to the $\mathrm{S}_{2}$ Electronic State Using a Realistic 24-Mode Model Hamiltonian,” J. Chem. Phys. 110, 936-946 (1999).

${ }^{57}$ G. A. Worth and L. S. Cederbaum, "Beyond Born-Oppenheimer: molecular dynamics through a conical intersection,” Annu. Rev. Phys. Chem. 55, 127-158 (2004).

${ }^{58} \mathrm{H}$. Wang and M. Thoss, "Multilayer Formulation of the Multiconfiguration Time-Dependent Hartree Theory,” J. Chem. Phys. 119, 1289-1299 (2003). 
${ }^{59}$ U. Manthe, “A Multilayer Multiconfigurational Time-Dependent Hartree Approach for Quantum Dynamics on General Potential Energy Surfaces," J. Chem. Phys. 128, 164116 (2008).

${ }^{60}$ O. Vendrell and H.-D. Meyer, "Multilayer Multiconfiguration Time-Dependent Hartree Method: Implementation and Applications to a Henon-Heiles Hamiltonian and to Pyrazine," J. Chem. Phys. 134, 044135 (2011).

${ }^{61}$ G. A. Worth, M. H. Beck, A. Jäckle, O. Vendrell, and H.-D. Meyer, The MCTDH Package, Version 8.2, (2000). H.-D. Meyer, Version 8.3 (2002), Version 8.4 (2007). O. Vendrell and H.-D. Meyer Version 8.5 (2013). Version 8.5 contains the ML-MCTDH algorithm. Current versions: 8.4.17 and 8.5.10 (2019). See http://mctdh.uni-hd.de/.

${ }^{62}$ J. Galego, F. J. Garcia-Vidal, and J. Feist, "Cavity-Induced Modifications of Molecular Structure in the Strong-Coupling Regime," Phys. Rev. X 5, 1-14 (2015).

${ }^{63}$ K. Bennett, M. Kowalewski, and S. Mukamel, "Novel Photochemistry of Molecular Polaritons in Optical Cavities," Faraday Discuss. 194, 259-282 (2016).

${ }^{64}$ M. Kowalewski, K. Bennett, and S. Mukamel, “Cavity Femtochemistry: Manipulating Nonadiabatic Dynamics at Avoided Crossings,” J. Phys. Chem. Lett. 7, 2050-2054 (2016).

${ }^{65}$ R. Islampour and M. Miralinaghi, “Theoretical Study of Internal Conversion Decay Rates Associated with the Three Lowest Singlet Electronic States in Pyrazine," J. Phys. Chem. A 113, 2340-2349 (2009).

${ }^{66}$ J. B. Pérez-Sánchez and J. Yuen-Zhou, "Polariton Assisted Down-Conversion of Photons via Nonadiabatic Molecular Dynamics: A Molecular Dynamical Casimir Effect,” J. Phys. Chem. Lett. 11, 152-159 (2020). 